\title{
Multiwavelength observations of a giant flare on CN Leonis ${ }^{\star} \star \star$ I. The chromosphere as seen in the optical spectra
}

\author{
B. Fuhrmeister ${ }^{1}$, C. Liefke ${ }^{1}$, J. H. M. M. Schmitt ${ }^{1}$, and A. Reiners ${ }^{2}$ \\ ${ }^{1}$ Hamburger Sternwarte, University of Hamburg, Gojenbergsweg 112, 21029 Hamburg, Germany \\ e-mail: bfuhrmeister@hs.uni-hamburg.de \\ ${ }^{2}$ University of Göttingen, Friedrich-Hundt-Platz 1, 37077 Göttingen, Germany
}

Received 10 January 2008 / Accepted 27 April 2008

ABSTRACT

\begin{abstract}
Aims. Flares on $\mathrm{dM}$ stars contain plasmas at very different temperatures and thus affect a wide wavelength range in the electromagnetic spectrum. While the coronal properties of flares are studied best in X-rays, the chromosphere of the star is observed best in the optical and ultraviolet ranges. Therefore, multiwavelength observations are essential to study flare properties throughout the atmosphere of a star.

Methods. We analysed simultaneous observations with UVES/VLT and XMM-Newton of the active M5.5 dwarf CN Leo (Gl 406) exhibiting a major flare. The optical data cover the wavelength range from 3000 to $10000 \AA$.

Results. From our optical data, we find an enormous wealth of chromospheric emission lines occurring throughout the spectrum. We identify a total of 1143 emission lines, out of which 154 are located in the red arm, increasing the number of observed emission lines in this red wavelength range by about a factor of 10 . Here we present an emission line list and a spectral atlas. We also find line asymmetries for H I, He I, and Ca II lines. For the last, this is the first observation of asymmetries due to a stellar flare. During the flare onset, there is additional flux found in the blue wing, while in the decay phase, additional flux is found in the red wing. We interpret both features as caused by mass motions. In addition to the lines, the flare manifests itself in the enhancement of the continuum throughout the whole spectrum, inverting the normal slope for the net flare spectrum.
\end{abstract}

Key words. stars: activity - stars: flare - stars: chromospheres - stars: late-type - stars: individual: CN Leo

\section{Introduction}

Flaring is a commonly observed phenomenon on late-type stars. During a flare event, large amounts of energy are released probably from magnetic field reconfigurations and emitted over a wide range of the electromagnetic spectrum. Although white light flares are relatively rare for the Sun, they are quite common among M dwarf stars, because of their lower photospheric background emission in the optical. Chromospheric emission lines react sensitively to flares in amplitude, line width, line shape, and wavelength shifts. In addition, optical flares often have X-ray counterparts, although there is no one-to-one relationship between X-ray and optical flares. During large flares, dM stars can show an increase in X-ray luminosity by factors up to 100 (e.g. Güdel et al. 2002, 2004), and even greater magnitude increases have occasionally been reported in the optical (e.g. Eason et al. 1992; Hawley \& Pettersen 1991).

The thermal properties of the coronal flare plasma can be diagnosed from the emitted soft X-ray emission, while gyrosynchrotron radio and microwave emission typically traces nonthermal particles in the corona. Additionally, emission lines

\footnotetext{
* Based on observations collected at the European Southern Observatory, Paranal, Chile, 077.D-0011(A) and on observations obtained with XMM-Newton, an ESA science mission with instruments and contributions directly funded by ESA Member States and NASA.

$\star \star$ Full Table 1 is only available in electronic form at the CDS via anonymous ftp to cdsarc.u-strasbg.fr (130.79.128.5) or via http://cdsweb.u-strasbg.fr/cgi-bin/qcat?J/A+A/487/293
}

in the ultraviolet mainly diagnose the transition region. Different wavelength ranges thus provide complementary information, so that multiwavelength observations of flares allow to study the contemporaneous reaction of different layers of the stellar atmosphere. A prototypical multiwavelength campaign was carried out by de Jager et al. (1989) for UV Cet, which was simultaneously observed in X-ray, optical, and radio. As a more recent example, Mitra-Kraev et al. (2005) observed a correlation between ultraviolet and X-ray flux for stellar flares. Another recent multiwavelength campaign was aimed at the flare star EV Lac and provided data in the radio, the optical, the ultraviolet, and in X-rays (Osten et al. 2005, 2006). These data cover a huge radio flare and a number of optical and X-ray flares. Osten et al. (2005) note that, for a flare in one wavelength region, counterparts in other wavelength regions are often missing. An example of a giant flare on AD Leo observed in the ultraviolet and optical is described by Hawley \& Pettersen (1991). AD Leo was again observed in multiwavelength by Hawley et al. (2003), where a number of medium-sized flares are covered and discussed. Smith et al. (2005) compare radio and X-ray flares on five active $\mathrm{M}$ dwarfs and provide possible mechanisms for uncorrelated flares. Multiwavelength observations also show that quiescent $\mathrm{X}$-ray, optical, and radio emission strengths are no longer correlated for late M and early L dwarfs (Berger et al. 2005, 2008a,b).

Our target star CN Leo (Gliese 406) is a well-known nearby flare star with a spectral type of M5.5 (Reid et al. 1995) or M6.0 (Kirkpatrick et al. 1991). Reiners et al. (2007) find a mean magnetic field of $B f \sim 2.2 \mathrm{kG}$, exhibiting night-to-night 
and, with less certainty, intra-night flux variations. CN Leo has previously been the target of a multiwavelength campaign in December 2005, covering the optical (VLT/UVES) and X-ray regimes (XMM-Newton; Fuhrmeister et al. 2007). During these observations, $\mathrm{CN}$ Leo once again proved to be quite active, showing two medium-sized and a number of smaller flares. Unfortunately, both larger flares occurred at the end of the optical observations and were therefore not properly covered. Now, CN Leo was again observed in the framework of a similar multiwavelength campaign, during which a spectacular flare occurred. This paper is the first in a series analysing the flare data, and it is structured as follows: in Sect. 2 we describe our observations obtained with UVES and XMM-Newton. In Sect. 3 we give an overview over the timing behaviour during the flare. The wide variety of chromospheric emission lines is reported in Sect. 4, while asymmetries found in the line shape of some emission lines are described in Sect. 5. A discussion and our conclusions are presented in Sect. 6.

\section{Observations and data analysis}

The multiwavelength observations reported in this paper were obtained simultaneously with XMM-Newton and ESO's Kueyen telescope equipped with the Ultraviolet-Visual Echelle Spectrograph (UVES) on three half nights in 2006: May, 19th/20th (first night), 21st/22nd (second night), and 23rd/24th) (third night). We describe the optical data of the first night here in detail, whereas the X-ray data will be described in Paper III of this series (Liefke et al. 2008).

UVES is a cross-dispersed echelle spectrograph that was operated using a dichroic beam splitter providing a blue arm spectrum (recorded with a single CCD) and a red arm spectrum (recorded with two CCDs) ${ }^{1}$. For the blue arm, a standard setup with central wavelength at $3460 \AA$ was used, while in the red arm a non-standard setup was used. The setup with central wavelength $8600 \AA$ with blue end at $6600 \AA$ was slightly blue-shifted to cover the $\mathrm{H} \alpha$ line. In our specific setup, used for the CN Leo observations presented here, the spectral coverage was between $3050 \AA$ to $3860 \AA$ in the blue arm and $6400 \AA$ to $10080 \AA$ in the red arm with a small gap from $8190 \AA$ to $8400 \AA$ due to the CCD mosaic. We used a slit width of $1^{\prime \prime}$ resulting in a resolution of $\sim 40000$. On May 19/20, the exposure times were $1000 \mathrm{~s}$ in the blue arm and $200 \mathrm{~s}$ in the red arm. In the three half-nights, our observations resulted in 68,24 , and 89 spectra in the red arm, and 16,4 , and 11 spectra in the blue arm.

The UVES spectra were reduced using the IDL-based REDUCE reduction package (Piskunov \& Valenti 2002). The wavelength calibration was carried out using Thorium-Argon spectra and resulted in an accuracy of $\sim 0.03 \AA$ in the blue arm and $\sim 0.05 \AA$ in the red arm. Absolute flux calibration was carried out using the UVES master response curves and extinction files provided by ESO. For fitting individual line profiles, we made use of the CORA program (Ness \& Wichmann 2002), that was originally designed for flux measurements of high-resolution $\mathrm{X}$-ray emission lines.

In addition, photometry with a time resolution of $\sim 1 \mathrm{~s}$ was obtained with the UVES exposuremeters, i.e., two blue and red photometers located in the two arms of the spectrograph. However, the exposuremeters are mainly used for calibration and engineering rather than for science, so they are not fluxcalibrated. We can nevertheless evaluate their spectral efficiency,

\footnotetext{
1 A detailed description of the UVES spectrograph is available under http://www. eso.org/instruments/uves/doc/
}

taking into account the efficiency curves of all components in the optical path (i.e. dicroic and pre-slit filter) as provided by $\mathrm{ESO}^{2}$, and assuming the quantum efficiency of a typical GaAS semiconductor device for the photometer itself. For our instrumental setup, the red exposuremeter should thus show a spectral efficiency similar to the Johnson $\mathrm{R}$ filter, covering the wavelength range from $\sim 5900-7100 \AA$, with its maximum at $6150 \AA$. The blue exposuremeter covers a broader wavelength range, from

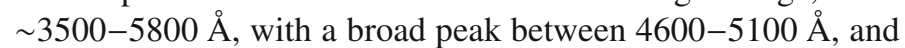
thus comprises a mixture of typical $B$ and $V$ filters.

The XMM-Newton satellite carries three co-aligned X-ray telescopes, each of which is equipped with one of the EPIC (European Photon Imaging Camera) MOS or PN instruments. These X-ray CCD detectors are photon-counting and thus provide timing information at the subsecond level and mediumresolution spectral resolution. Two of the X-ray telescopes are additionally equipped with Reflection Grating Spectrometers (RGS) that provide high-resolution X-ray spectroscopy. The $\mathrm{X}$-ray instruments are accompanied by the Optical Monitor OM, an optical/UV telescope that can be used with different filters in imaging or fast readout mode; the latter mode provides photometry with a time resolution of $\sim 1 \mathrm{~s}$. All instruments are normally operated simultaneously ${ }^{3}$.

Some of the X-ray data has already been published (Schmitt et al. 2008), and we will give a detailed analysis of the X-ray data in a subsequent paper (Liefke et al. 2008). In this paper we focus on the Optical Monitor, which was operated in fast mode with the U-band filter covering wavelengths between 3100-3900 ^, perfectly matching the spectral range of the blue arm UVES spectra. The observations are split into several exposures, each with an exposure time of $3200 \mathrm{~s}$ with gaps of about $300 \mathrm{~s}$ in between. We analysed the XMM-Newton data with the OM packages of the XMM-Newton Science Analysis System (SAS) software, version 7.0.

\section{Time resolved spectral response of the flare}

At the beginning of the first night of our observing campaign, a giant flare occurred; the onset properties of this flare have been described in detail by Schmitt et al. (2008). The corresponding red, blue and the $U$-band lightcurves as derived from the two UVES exposuremeters and the OM, are shown in Fig. 1, where we also show the start times of the blue and red spectra. Note that the spectra in the blue and red arms never start at the same time. Besides this large flare, several weaker flares occurred during the three half nights, which will be discussed in a separate publication. In this paper we concentrate on the emission observed from the giant flare.

The flare onset at UT 23:46:30 is seen as an enormous intensity increase in all wavelength bands, i.e. in X-rays, in the $\mathrm{U}$ band, as measured with the OM, and with the blue and red UVES exposuremeters. During the first few seconds of the flare, a sharp peak in the optical and in soft X-ray occurs. Schmitt et al. (2008) interpret this as the signature of a coronal explosion. After the first sharp peak, a broader peak is visible in the optical UVES exposuremeter data, followed by an even broader maximum in X-rays. The quasi-quiescent level after the flare is 8 percent higher than the pre-flare level in the blue arm and

\footnotetext{
2 http://www . eso.org/instruments/uves/tools/

3 More details on the instruments onboard XMM-Newton can be found in the XMM-Newton Users' Handbook, available at http://xmm. vilspa.esa.es/external/xmm_user_support/documentation/ uhb/index.html
} 


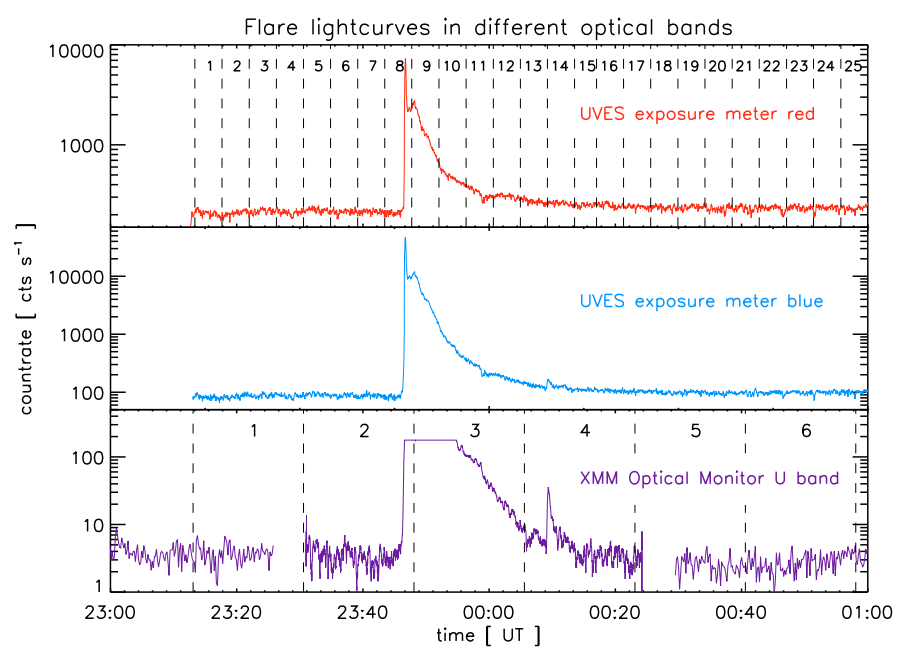

Fig. 1. Optical lightcurves of the large flare on CN Leo. Top: UVES red arm exposuremeter. Middle: UVES blue arm exposuremeter. Bottom: $X M M$ Optical Monitor (cutted above count rates $>200 \mathrm{cts} \mathrm{s}^{-1}$ ). The vertical dashed lines in the red and $U$ band plots mark the start times of the red and blue UVES spectra, respectively.

6 percent in the red arm. Also, the chromospheric emission lines remain enhanced after the flare. A very similar time response of a large flare on UV Cet with a sharp first peak, a broad second peak, and the quiescent level after the flare higher than before was described by de Jager et al. (1989).

At the first flare peak, the flux increased by a factor of about 550 in the blue UVES arm, and by about 40 in the red arm compared to the pre-flare level. During the whole flare peak, the Optical Monitor entered saturation, i.e. the count rate in the $U$ band exceeded the valid range for a reliable deadtime correction and flux conversion so that no reliable count rates can be derived. Additionally, the source extraction window suffers extremely from the so-called modulo- 8 readout pattern, which disturbs the PSF of the OM. We thus do not consider the reconstructed shape of the lightcurve as trustworthy for count rates $>200 \mathrm{cts} \mathrm{s}^{-1}$ and cut the lightcurve for count rate values exceeding this number.

The blue and red exposuremeter lightcurves are quite similar, and the decay after the broad second maximum is rather fast. In contrast, the decay time of the $U$-band lightcurve is much longer. Since the wavelength ranges covered by the exposuremeters contain far fewer chromospheric emission lines than the $U$-band, they mainly reflect the behaviour of the continuum, while the chromospheric emission lines dominate $U$-band lightcurve during the decay phase.

\subsection{Blue part of the spectrum}

In the blue arm, the large flare is covered by spectra Nos. 2-4. They are shown, together with the quiescent spectrum No. 1, in Fig. 2. The last $\approx 60$ s of exposure No. 2, taken from UT 23:30:46 to $23: 47: 26$, cover the impulsive phase of the flare; the contribution of quiescent emission in exposure No. 2 should be negligible. The spectrum is dominated by blackbody emission (see Sect. 3.3). The Balmer lines have gained in amplitude and developed very broad wings. Many other chromospheric emission lines start to show up as well, but they are totally outshone by the blackbody radiation during the first $\approx 60 \mathrm{~s}$ of the flare. Therefore, the true wealth of emission lines becomes visible only in spectrum No. 3, covering the broader peak of the flare from 23:48:16 to 00:04:56. In spectrum No. 4, most of the emission lines show still increased emission, while in spectrum No. 5, a new quiescent level is reached. Simultaneously, the flux reaches the quiescent level around UT 00:30 as shown in the lightcurve in Fig. 1. A detailed example of the evolution of a typical part of the blue spectrum covering the $\mathrm{H}_{9}$ line is shown in Fig. 3. An atlas of spectrum No. 3, which was used to create an emission line list, can be found in Figs. 4 and 5.

\subsection{Red part of the spectrum}

In the red arm, the very first seconds of the flare are covered by spectrum No. 8, taken from UT 23:43:20 to UT 23:46:40. In this spectrum, a slightly higher level of continuum emission is already visible, especially in the bluer part of the spectrum around $\mathrm{H} \alpha$. Some lines, e.g. the He I line at $6678 \AA$, are starting to go into emission, while others are not. This should partly be due to a lack of sensitivity since only a rather short time interval of the flare is covered, which prevents weak lines from showing up against the continuum. However, this may also be due to nonequilibrium conditions during the first few seconds of the flare, since lines formed at different atmospheric depths should react on different timescales.

The consecutive spectrum No. 9 starts at UT 23:47:37. Here, the continuum enhancement reaches a maximum and is clearly seen throughout the whole red spectrum. The net flare spectrum, i.e. with the quiescent flux subtracted, clearly shows an inverse slope with the flux increasing towards the blue end. This effect has already been described for a similar wavelength range for flares on 2MASS J0149090+295613 (M9.5, Liebert et al. 1999) and 2MASS J1028404-143843 (M7, Schmidt et al. 2007). Moreover, spectrum No. 9 shows a wide variety of emission lines never observed before in this spectral range. We show the flare spectrum No. 9 with spectrum No. 1 subtracted in Fig. 6, and the two spectra themselves in Fig. 7.

The emission lines decay on very different timescales. For example, emission of the Ca II triplet lines decays very slowly and can be noted until spectrum No. 16, while the emission core of $\mathrm{K} \mathrm{I}$ is undetectable after spectrum No. 11. The same applies to the net flux of the three O I lines around $7772 \AA$, while the three O I lines around $9262 \AA$ are only found in the flare spectrum No. 9. The timing behaviour of the line flux is shown in Fig. 8 for some important emission lines.

The continuum emission stays enhanced until about UT 00:15 (spectrum No. 15) in the blue part of the red arm, while the red part of the red arm is only elevated in spectra Nos. 8-10.

\subsection{Temperature evolution}

The well-defined slope of the flare continuum in our spectra suggests a blackbody origin. We fitted temperature and emitting area to the smoothed blue spectra Nos. 2 and 3 after removing the emission lines. In both cases, the contribution of the quiescent atmosphere was neglected. The fit gives temperatures of $\sim 11300 \mathrm{~K}$ for spectrum No. 2 (also discussed in Schmitt et al. 2008) and of $\sim 9100 \mathrm{~K}$ for spectrum No. 3; however these temperatures correspond to averaged values during the exposure (i.e. for the flare onset during the first minute of the flare in spectrum No. 2, and over the whole exposure time of $1000 \mathrm{~s}$ during the broader peak in spectrum No. 3), since the thermal continuum emission can be expected to undergo a rapid decrease in temperature and flux. Figure 2 shows the two best-fit blackbodies as straight (red) lines; blackbody fits with fixed temperatures 


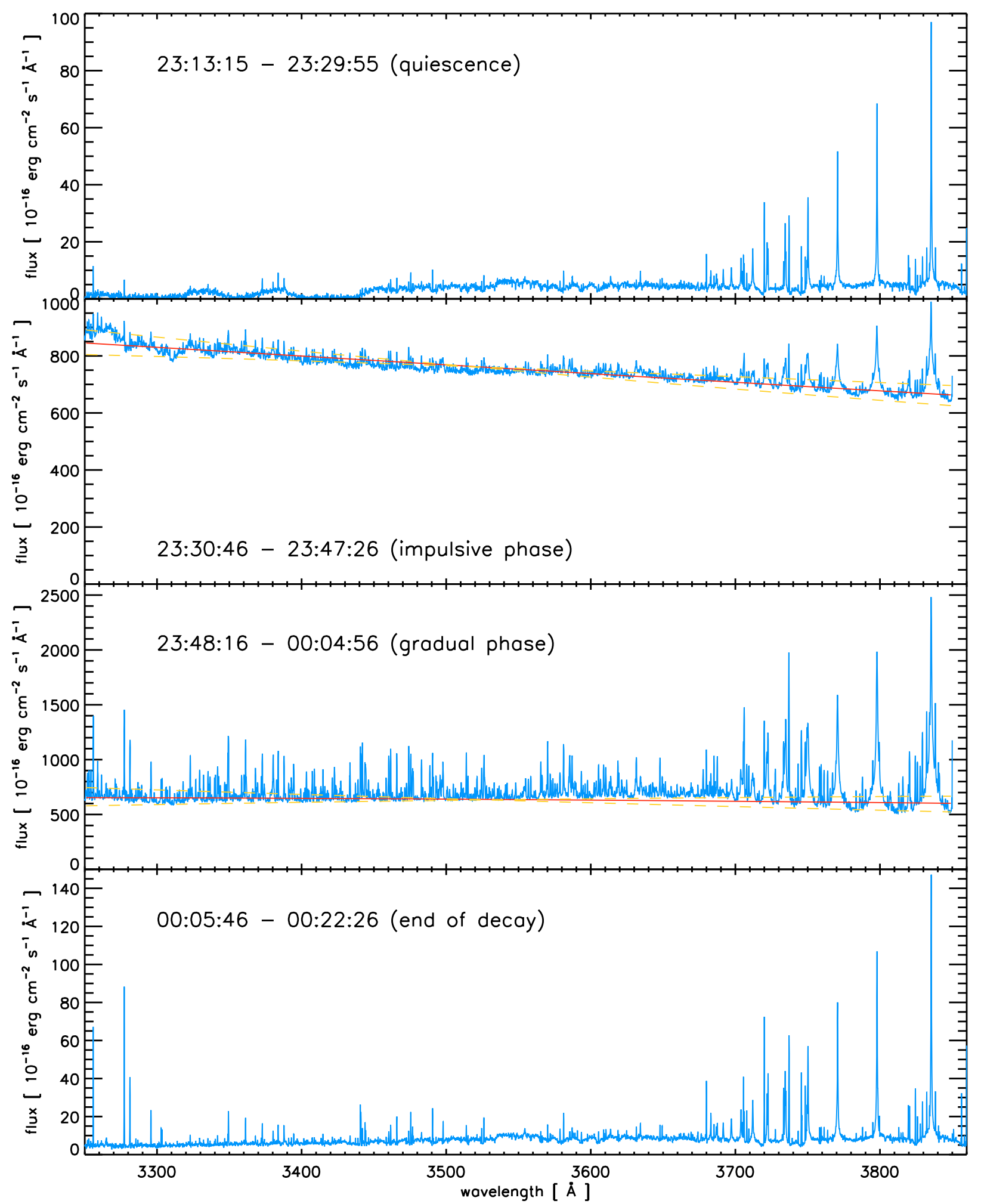

Fig. 2. Sequence of blue UVES spectra Nos. 1 to 4 covering the flare (top to bottom). Note the different flux scales. Overlaid in red and orange are different blackbody fits.

of $10000 \mathrm{~K}$ and $15000 \mathrm{~K}$ in spectrum No. 2 and with $7000 \mathrm{~K}$ and $10000 \mathrm{~K}$ in spectrum No. 3 are also shown for comparison as dashed (orange) lines. Thus the uncertainties of the temperature fit can be estimated not to exceed $1000 \mathrm{~K}$. The resulting sizes of the emitting area are then $1-10 \times 10^{18} \mathrm{~cm}^{2}$. In spectrum No. 4 , the continuum level has nearly decreased to quiescent levels.
The same procedure can be applied to the red arm spectra Nos. 8-10, with the quiescent flux subtracted. Since for temperatures around $10000 \mathrm{~K}$ the wavelength range covered by the red arm spectra is located far in the Rayleigh-Jeans part of the blackbody spectrum, the derived temperatures have rather large errors, which we estimate to reach up to $5000 \mathrm{~K}$. Additionally, the true continuum level at the position of the strong telluric 


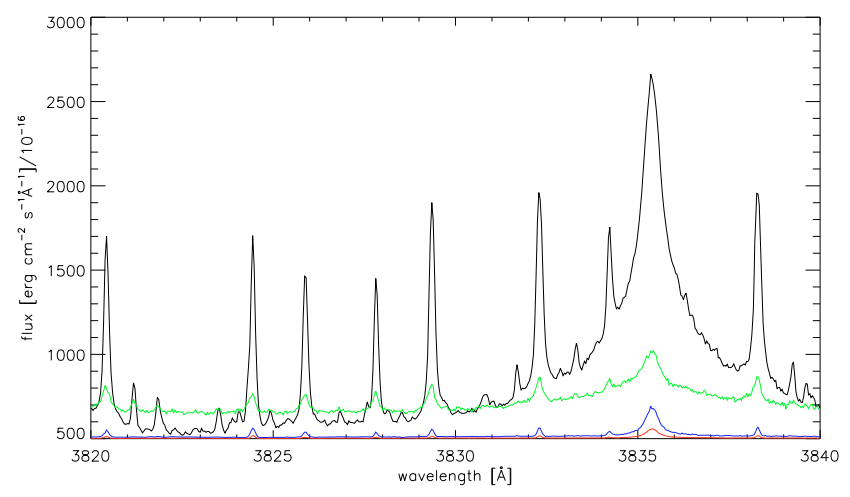

Fig. 3. Spectral region around the Balmer line $\mathrm{H} 9$ in the blue arm. The evolution of the hydrogen and of several metal lines can be seen. The blue/lowest line denotes the pre-flare spectrum (No. 1). The green/light grey line denotes the spectrum covering flare onset (No. 2). The black line and the red/grey line denote the two following spectra (Nos. 3 and 4). The black spectrum (No. 3) was used for the line identification.

absorption bands is difficult to assess. It is thus appropriate to restrict the analysis to the short-wavelength part of the spectrum. The red flare spectrum No. 8, covering the impulsive phase of the flare, can be fitted with a temperature of $\sim 27700 \mathrm{~K}$ (and with $\sim 19600 \mathrm{~K}$ considering both parts of the red arm). For the red spectrum No. 9 we determined a temperature of $\sim 5600 \mathrm{~K}$. Spectrum No. 10 gives temperatures of 2600 to 3200 K, i.e. the flare plasma has cooled to CN Leo's quiescent photospheric temperature of $\approx 2900 \mathrm{~K}$ (Pavlenko et al. 2006; Fuhrmeister et al. 2005b). The values derived for the emitting area are consistent with those from the blue spectra alone.

Putting the derived temperatures in the right chronological order, one starts with red spectrum No. 8 (covering the first few second of the flare) with probably more than $20000 \mathrm{~K}$, then followed by the blue spectrum No. 2 (covering the first minute) with about $11000 \mathrm{~K}$. In the subsequent blue spectrum No. 3 and red spectrum No. 9, the measured temperature is rapidly dropping from $9100 \mathrm{~K}$ to $5600 \mathrm{~K}$, reaching the quiescent photospheric temperature for red spectrum No. 10.

The optical flare continuum emission can most likely be attributed to photospheric plasma that is reprocessing UV and EUV line emission, which itself was induced by heating from nonthermal particles (as discussed by Hawley \& Fisher 1992, where they confirm the blackbody hypothesis from photometric UV observations of flares on AD Leo). Several spectra covering flares on late $\mathrm{M}$ dwarfs can be found in the literature; however, they typically cover only the spectral range of $\mathrm{H} \alpha$ and longwards in wavelength, introducing large uncertainties into a possible blackbody fit. Our blue spectra allow us to assess the blackbody temperature from the slope of the spectra with reasonably small uncertainties. Together with the different temporal coverage of the flare in the red arm, we can conclude that the plasma is initially heated to temperatures probably greater than $20000 \mathrm{~K}$ and cooling rapidly afterwards, reaching already the photospheric level when the chromospheric line emission is still seen near its maximum.

\section{Identification of chromospheric emission lines}

For the flare spectra, we point out the wealth of chromospheric emission lines. Because many of the lines, especially in the red part of the spectrum, were not observed in emission before, we provide an emission line list for the flare spectrum.

\subsection{General information on the line catalogue}

In the blue spectrum No. 3 and the red spectrum No. 9, we detected 1143 chromospheric emission lines altogether, with 989 out of them located in the blue arm and 154 in the red arm. Out of the 154 emission lines in the red arm spectrum, 110 were analysed in the flare-only spectrum. While the blue arm typically comprises pure emission lines, emission cores in absorption lines and filled in absorption lines are also found in the red arm. From our red spectra, we show the Paschen 7 line (pure emission) in the flare spectra (Fig. 9), the K I line (emission core) at $7699 \AA$ (Fig. 10), and the Na I line (filled in absorption) at $8183 \AA$ (Fig. 11) as three representative examples of each type of enhanced flare emission.

To determine the line parameters we used an IDL routine that automatically fits background, central wavelength, $F W H M$, and line flux as free parameters using a Gaussian parameterisation for every manually indicated emission line in a certain wavelength range. We typically chose $10 \AA$ as the wavelength range for one fitting process, since the background variations on such an short wavelength interval are acceptable. The applied program was kindly provided to us by Dr. Jan-Uwe Ness. The line fit parameters including a (possible) identification can be found in Table 1.

In the line catalogue we provide the measured emission line fluxes. These may be affected by rather large errors mainly for two reasons. First, the description with a Gaussian may result in a poor fit if the lines have broad wings. The 18 affected lines belong to the ions H I, O I, Fe I, and He II and are flagged accordingly. Second, the background/quasi-continuum may be somewhat ill-defined. This is true for emission lines in a broader emission line wing (stated in the remarks), but also for emission cores of absorption lines and for varying background, with the latter found especially in the red arm due to molecules. Also, the $\mathrm{H} \alpha$ line and the Ca II triplet lines are saturated in the peak flare spectrum, which was used for the identification list. Moreover, 5 Balmer/Paschen lines could not be fitted at all due to their large width (but they are clearly present). Therefore, individual lines may have flux errors larger than a factor of 2 , but normally the flux measurements should have errors below 30 percent.

We mainly used the Moore catalogue (Moore 1972) for identifying the lines, a few lines were identified using the NIST database $^{4}$. For the identifications from the Moore catalogue, the multiplet is also given in the catalogue. The spectra were first shifted to the rest wavelength for the identification process. This enables us to find systematic lineshifts caused by the flare, which was not the case in general. The average displacement between measured central wavelength and laboratory wavelength is $0.01 \AA$ in the blue arm and $0.05 \AA$ in the red arm.

We were not able to identify 7 emission lines. Another 167 (15\%) lines have only tentative identifications for one of the following reasons: (1) the line was not found in the Moore catalogue, (2) the wavelength shift to the possible laboratory wavelength is large, (3) the line is severely blended with other lines, and (4) (most often) the line is the only one out of the multiplet. However, there are few lines, which are single identifications out of a multiplet or are from a singlet, that are flagged as

\footnotetext{
4 Available online under

http://physics.nist.gov/PhysRefData/ASD/index.html
} 


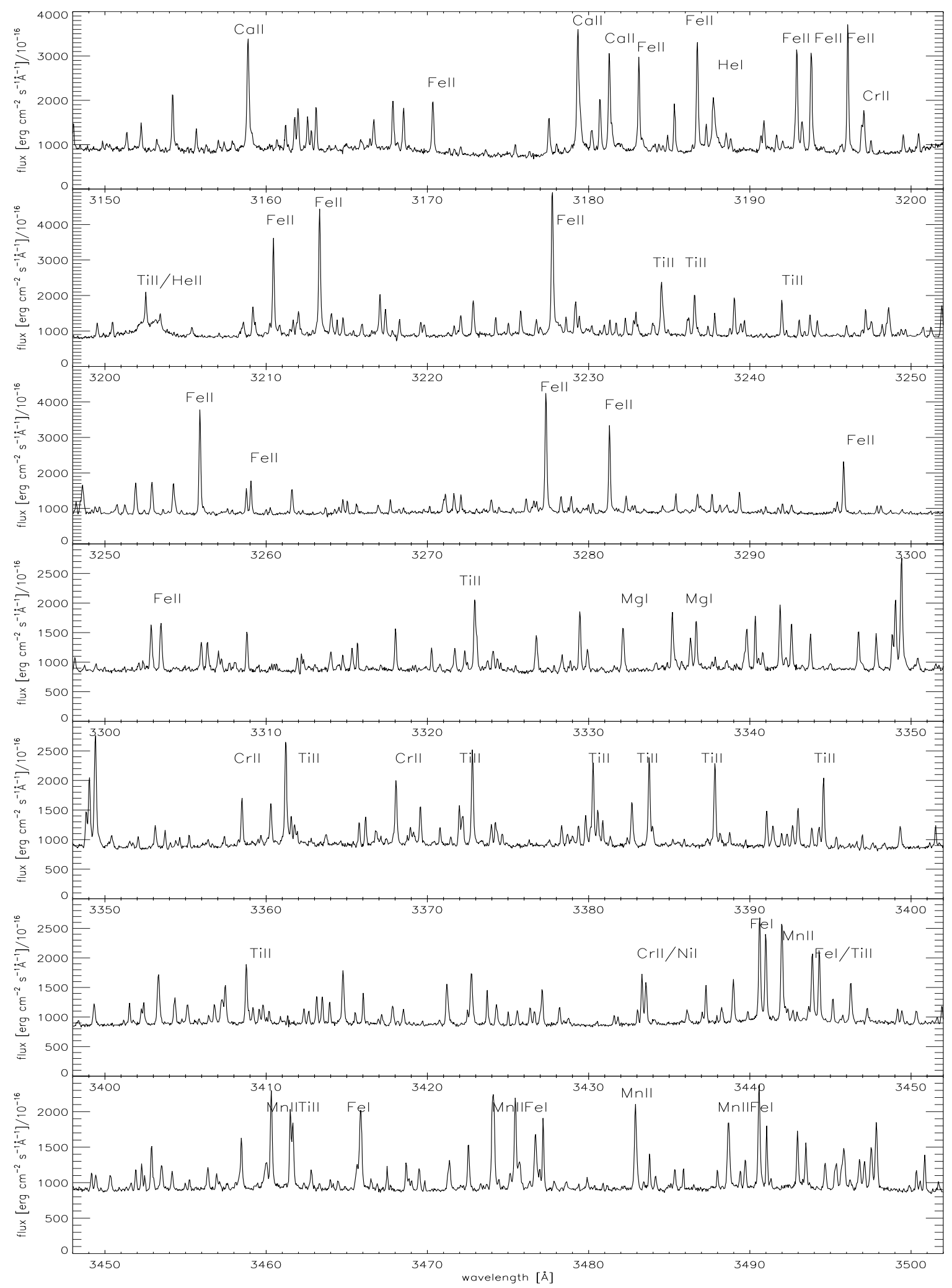

Fig. 4. Spectral atlas of blue spectrum No. 3 from 3150 to $3500 \AA$ A. Some important emission lines are identified, for other identifications see the emission line list.

secure identifications. The catalogue includes a set of flags providing information on the identification status of the line. If there are other possible identifications for the line, these are normally given as remarks, even if the line is identified securely. The latter may be the case if the line belongs to a multiplet out of which a number of lines are already found, but there are other (plausible) identifications with identical or very near laboratory wavelength.

Since we excluded doubtful features from our line list, the list cannot be claimed to be complete, especially for the weak lines with fluxes below $5 \times 10^{-14} \mathrm{erg} \mathrm{s}^{-1} \mathrm{~cm}^{-2}$. Also, we partly 


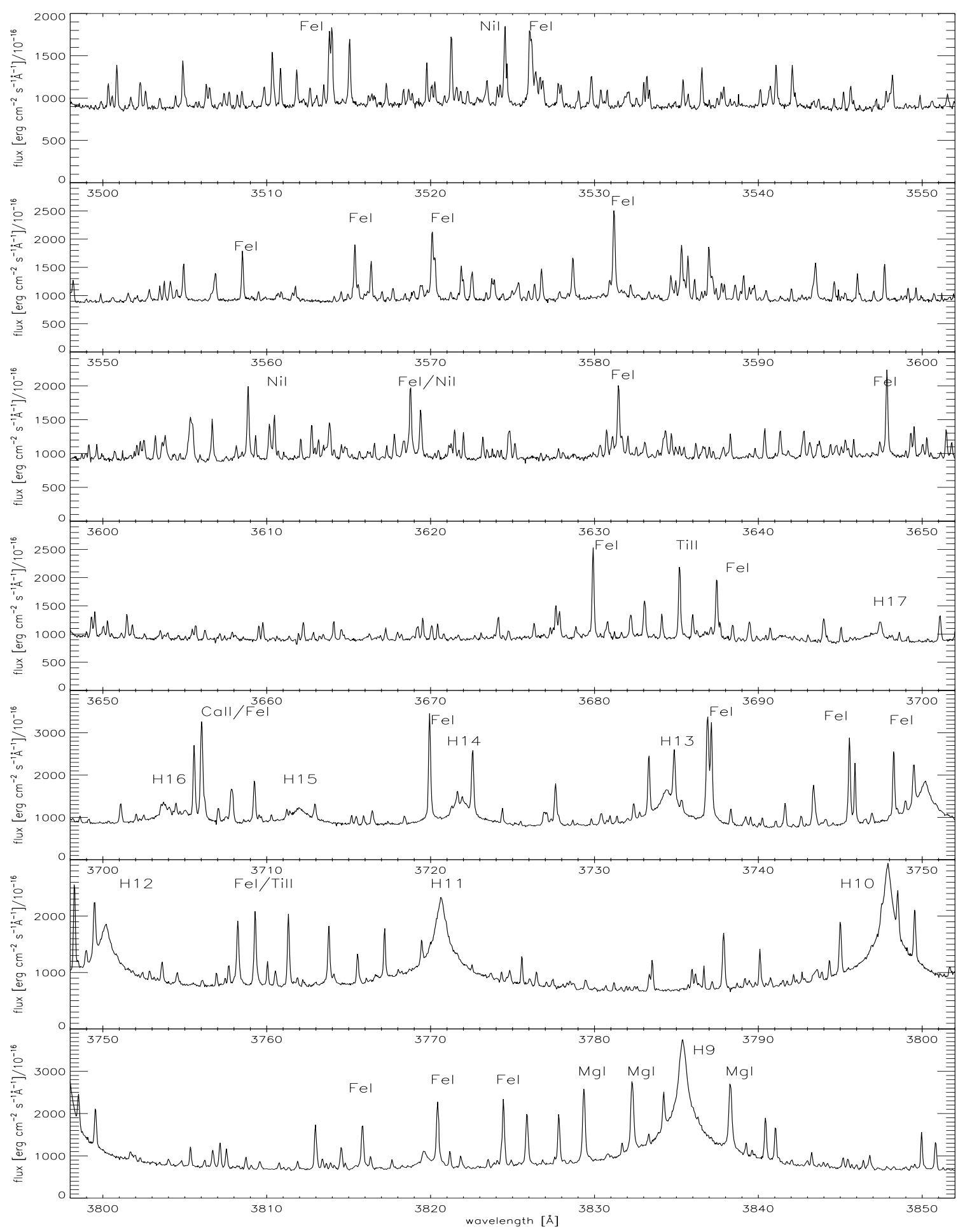

Fig. 5. Same as Fig. 4 from 3500 to $3850 \AA$.

used the flare-only spectrum in the red arm, as denoted by the identification flag for individual lines. To create this flare spectrum, we subtracted the first spectrum of the May 19/20 night, which shows CN Leo in quiescence.

The identified lines belong to 35 different ions in total, and the basic statistics of these flare lines can be found in Table 2. For the appearance of V II lines in emission, we are not aware of any description of V II chromospheric emission lines for flare stars in the literature.

\subsection{Comparison to other work}

Many of the emission lines in the flare-only spectrum (with the quiescent spectrum subtracted) have not been observed before. Flare stars are traditionally observed in the ultraviolet and $\mathrm{X}$-ray regime, rather than at these red wavelengths. In addition, observations in this wavelength range often lack the resolution necessary for resolving the metal emission lines without wings. Nevertheless, most of the stronger emission lines like the Paschen lines, the He I, the Ca II lines, and others have been 


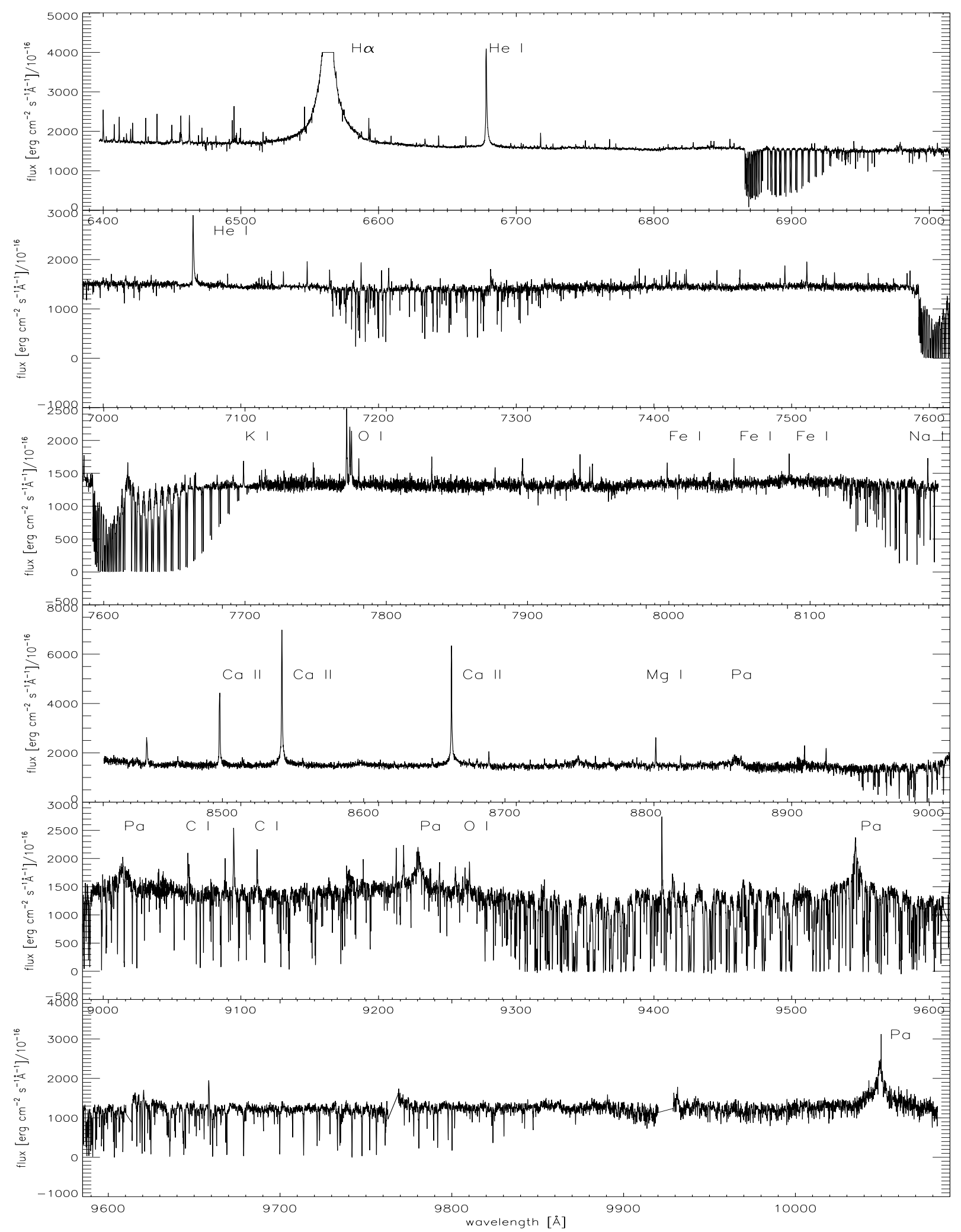

Fig. 6. Difference (flare-only) spectrum of the red arm mosaic of spectrum Nos. 9 and 1 (same as in Fig. 7). A wide variety of emission lines can be noted, which is totally atypical for this wavelength range. Also the slope is inverted compared to the quiescent spectrum.

described in spectacular flares on very late-type $\mathrm{M}$ dwarfs. For example, 2MASS J0149090+295613 (spectral type M9.5 V) was observed by Liebert et al. (1999) during a flare showing 16 emission lines in the red part of the spectrum among them 5 Paschen lines and the Ca II triplet. Schmidt et al. (2007) also describes two flare events on LP 412-31 (M8) and 2MASS J1028404-143843 (M7), showing emission lines in the considered wavelength range. Both stars show the Ca II triplet 

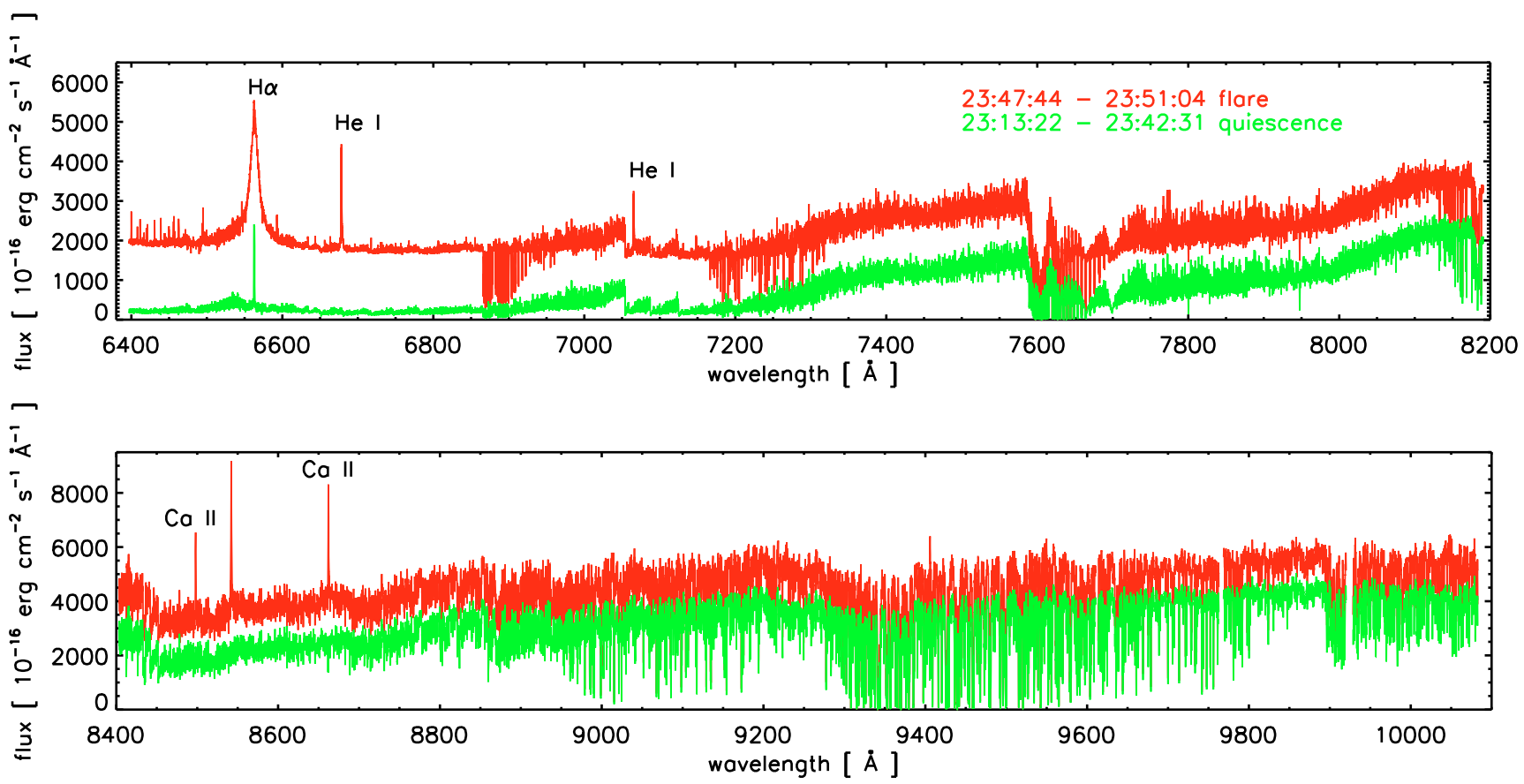

Fig. 7. UVES red spectra in quiescence (spectrum No. 1 in green/grey) and in flare (spectrum No. 9 in red/black).

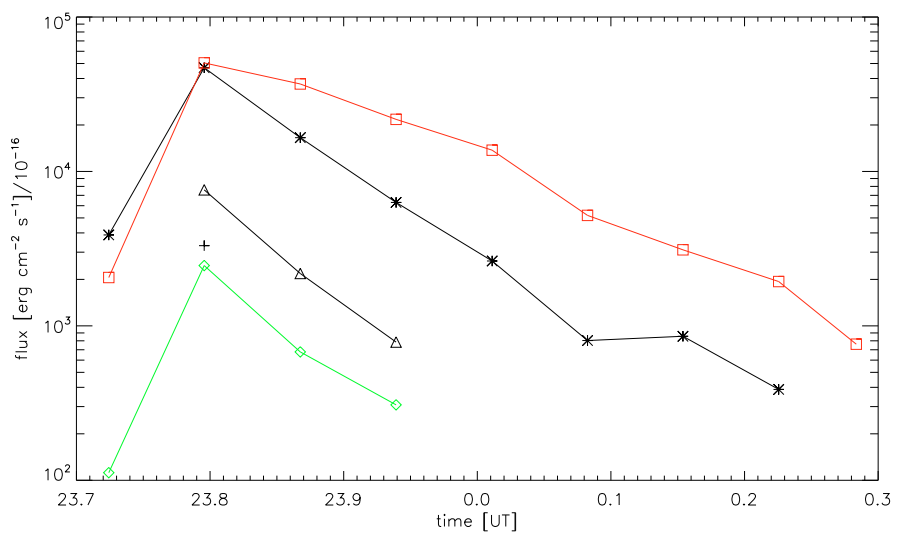

Fig. 8. Timing behaviour of line fluxes with quiescent flux removed for some important emission lines. Red squares denote the Ca II line at $8542 \AA$ (narrow and broad component; line core is saturated during flare peak), black asterisks denote the He I line at $6678 \AA$ (narrow and broad component), black triangles denote the O I line at $7774 \AA$, green diamonds denote the emission core of the K I line at $7699 \AA$, and the black cross the line flux of the Na I line, which is elevated only during flare peak.

and hydrogen Paschen emission up to $P_{11}$. During all these three flares, the Paschen lines are much more prominent than in our case. We ascribe this to the earlier spectral type of CN Leo, which has more continuum flux at these wavelengths.

As another example we compare our observations to flares on the old inactive M4 star Gliese 699 (Barnard's star) by Paulson et al. (2006). There, the wavelength coverage was from 3700 to $10800 \AA$, with a spectral resolution of $\sim 60000$. Paulson et al. (2006) have also produced an emission line list. In the overlapping wavelength range with our blue arm (from 3730 to $3860 \AA$ ) they find 28 lines, where we find 135 emission lines. Among these, there is one line at $3772 \AA$ where we located Ni I at $3772.530 \AA$, while Paulson et al. (2006) identified Fe I at

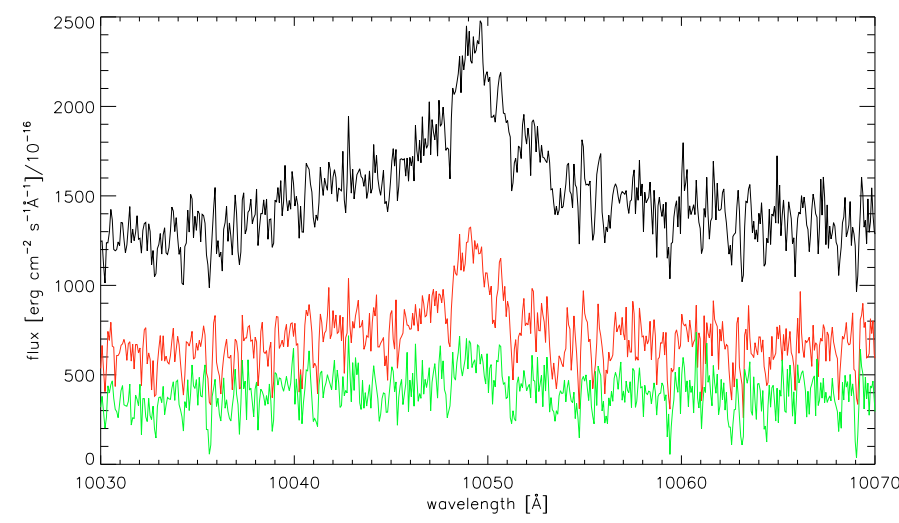

Fig. 9. Paschen (P7) line at $10049 \AA$ with a cosmic removed at $10049.972 \AA$. The black line corresponds to the red flare-only spectrum No. 9. Also shown are the two consecutive flare-only spectra, where the line quickly decays.

$3772.23 \AA$. With the exception of this one line, we found all lines also identified by Paulson et al. (2006) in the blue wavelength range. In the red arm, we found 154 lines, while Paulson et al. (2006) detected 11 enhanced lines redward of $6400 \AA$. Again, we were able to locate all of their lines with one discrepancy at $6462 \AA$, where we found a line at $6462.566 \AA$ tentatively identified as Ca I, while Paulson et al. (2006) found an enhanced line at $6462.73 \AA$ identified as an Fe I line. It is not clear why we do not see these two Fe I lines identified for Barnard's star. Our resolution is sufficient to detect these two lines and not to confuse them with our additional detections.

\subsubsection{He II emission from the transition region}

Among the chromospheric emission lines, there is an He II line at $3203.104 \AA$ originating from the transition region. This line, which is barely recognisable outside of flares, reacts to the flare as well, but not as strong and fast as the chromospheric lines. 
Table 1. First 5 rows of the line catalogue. The whole table is accessible electronically at the CDS.

\begin{tabular}{cccccrcc}
\hline \hline $\begin{array}{c}\text { Central wavelength } \\
{[\AA]}\end{array}$ & $\begin{array}{c}F W H M \\
{[\AA]}\end{array}$ & $\begin{array}{c}\text { Flux } \\
{\left[\mathrm{erg} \mathrm{s}^{-1} \mathrm{~cm}^{-2}\right]}\end{array}$ & $\begin{array}{c}\text { Catalogued wavelength } \\
{[\AA]}\end{array}$ & Ion & $\begin{array}{c}\text { Multiplet } \\
\text { id flag }\end{array}$ & Comment \\
\hline 3057.45 & 0.057 & $4.43 \mathrm{e}-13$ & 3057.446 & $\mathrm{Fe} \mathrm{I}$ & 28 & 0 & $n$ \\
3059.09 & 0.057 & $4.27 \mathrm{e}-13$ & 3059.086 & $\mathrm{Fe} \mathrm{I}$ & 9 & 1 & $n$ \\
3062.26 & 0.045 & $2.08 \mathrm{e}-13$ & 3062.234 & Fe II & 108 & 0 & $n$ \\
3064.62 & 0.042 & $9.17 \mathrm{e}-14$ & 3064.623 & $\mathrm{Ni} \mathrm{I}$ & 26 & 0 & $n$ \\
3065.32 & 0.042 & $1.34 \mathrm{e}-13$ & 3065.315 & Fe II & 97 & 0 & $n$ \\
\hline
\end{tabular}

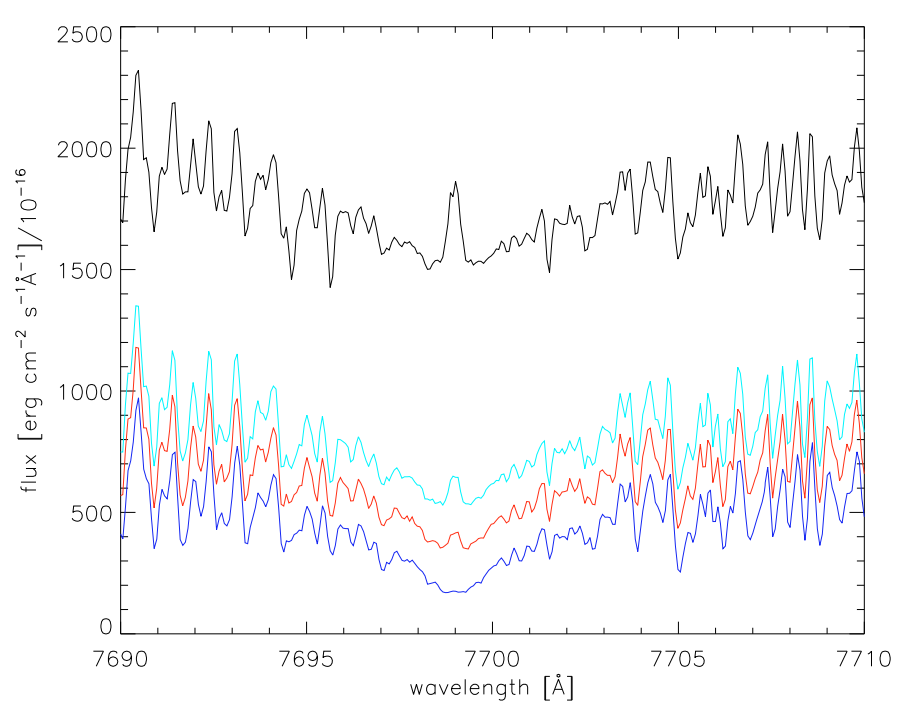

Fig. 10. KI line at $7699 \AA$. The quiescent spectrum No. 1 is shown in blue (lowest line). The black line denotes the flare spectrum No. 9, the turquoise/light grey and red/grey lines denote the two following spectra. Note the emission core during the flare.

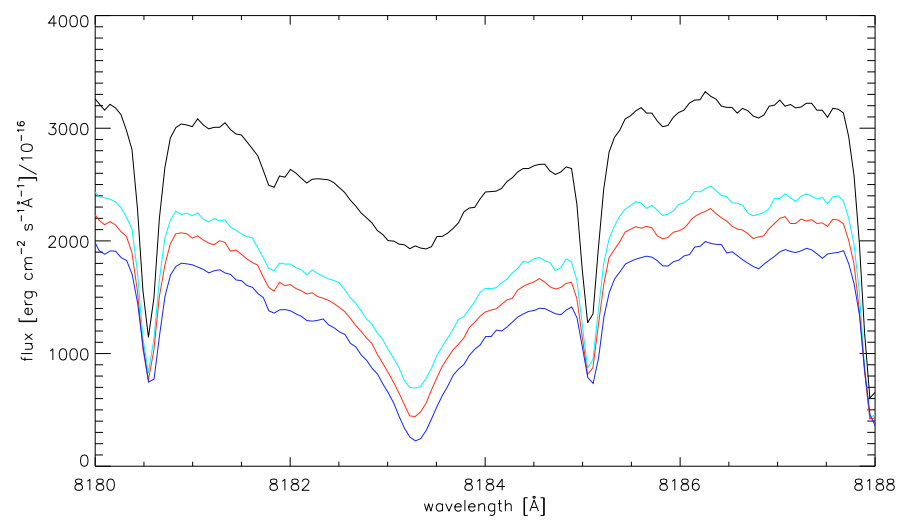

Fig. 11. Na I line at $8183 \AA$ A. The quiescent spectrum No. 1 is shown in blue/lowest line. Colour coding as in Fig. 10. In contrast to the KI line in Fig. 10, no emission core is seen, but the line fills in with chromospheric emission, resulting in an emission line in the flare-only spectra.

During the flare peak in spectrum No. 3, the He II line is not as strong as the two flanking chromospheric Ti II emission lines. In the following spectrum No. 4, the two Ti II lines have completely decayed, while the He II line is still present. As found by Fuhrmeister et al. (2007), the situation is quite different during the flare onset of 13 December 2005 as seen in spectrum No. 12. There, the He II line reacts much more strongly than the two Ti II lines, which may be interpreted as due to different energy deposition heights for the two flares. The strong flare discussed here had an energy deposition into very deep layers (as can also be seen from the strong blackbody/photospheric reaction and the
Table 2. Chromospheric emission line identification.

\begin{tabular}{ll|ll}
\hline \hline Ion & No. lines & Ion & No. lines \\
\hline H I & $15(15)$ & Mg I & $13(11)$ \\
He I & $7(5)$ & Mg II & $5(3)$ \\
He II & $1(1)$ & Na I & $2(1)$ \\
Sc II & $16(16)$ & Na II & 0 \\
Ti I & $15(6)$ & K I & $1(0)$ \\
Ti II & $125(116)$ & Ca I & $13(11)$ \\
V II & $32(30)$ & Ca II & $9(8)$ \\
Cr I & $16(12)$ & C I & $5(5)$ \\
Cr II & $72(59)$ & N I & $1(0)$ \\
Mn I & $13(11)$ & O I & $6(6)$ \\
Mn II & $9(8)$ & Ne I & $1(0)$ \\
Fe I & $494(428)$ & S I & $2(0)$ \\
Fe II & $90(70)$ & Al I & $4(2)$ \\
Co I & $47(43)$ & Si I & $10(3)$ \\
Co II & $1(1)$ & Si II & $1(0)$ \\
Ni I & $98(87)$ & no id & 7 \\
Ni II & $5(3)$ & & \\
Cu I & $3(2)$ & & \\
Ba II & $1(1)$ & & \\
Y II & $1(0)$ & & \\
Zr II & $2(2)$ & & \\
\hline
\end{tabular}

${ }^{1}$ In parenthesis the number of secure identifications.

enormous width of the Balmer series lines). Thus, the transition region seems to be heated more gently, but of course this is difficult to decide from only one line.

\section{Line asymmetries}

Line asymmetries during flares have been described at various times for different emission lines. Blue excess-line emission was described for example by Gunn et al. (1994) for a flare on AT Mic, while Crespo-Chacón et al. (2006) note red line asymmetries in the Balmer lines during various flares on $\mathrm{AD}$ Leo $(\mathrm{M} 3 \mathrm{~V})$. The latter found the asymmetries stronger for larger flares and stronger for higher members of the Balmer series. They also analysed the $\mathrm{Ca} \mathrm{II} \mathrm{H}$ and $\mathrm{K}$ lines, but did not find any asymmetry. Also, Fuhrmeister et al. (2005a) found red wing asymmetries in the Balmer and $\mathrm{He} \mathrm{I}$ lines for a long duration flare on LHS 2034 (M6V). The additional red wing flux decayed faster for the Balmer lines than the line flux. For the He I lines, the flux creating the asymmetry first increased relative to the line flux and then decayed; examples of papers describing line asymmetries are given by these authors.

Additional blue wing flux is normally interpreted as upmoving material, which is expected for stellar flares, while additional red wing flux has been ascribed mostly to downflows during the flares. For the Sun, similar mass flows (chromospheric downward condensations) are known (Canfield et al. 1990) to last for a few minutes. Since the asymmetries in $\mathrm{M}$ dwarfs often persist much longer, various flare kernels producing several 

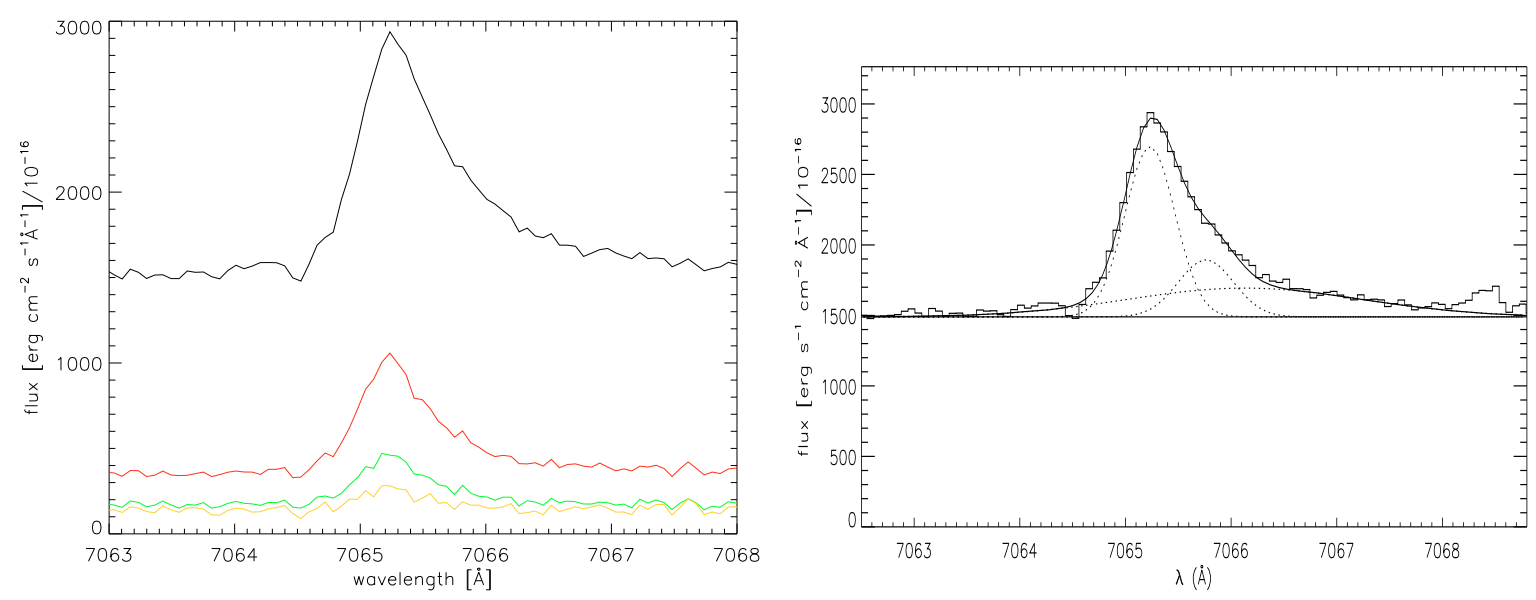

Fig. 12. Red-wing asymmetry for the He I line at 7065.30/7065.93 A. Left: flare spectrum No. 9 of the line and the three consecutive spectra (with the quiescent spectrum subtracted for each spectrum). Right: three-component Gaussian fit of the line with CORA. The asymmetry manifests itself in the broad Gaussian component. The background has been fitted manually.

downflows have been proposed (Doyle et al. 1988; Fuhrmeister et al. 2005a).

In the present data, line asymmetries have also been found. Besides asymmetries in the Balmer and He I lines, asymmetries for Ca II lines have been found for the first time. Asymmetries may be present in the Mg II lines, but they are all very weak, so that a red wing is hard to distinguish from the noise/molecular lines.

In the following we give a detailed presentation of the line asymmetries.

\subsection{Hel lines}

Seven He I lines are observed in the flare peak spectra Nos. 9 (red arm) and 3 (blue arm). Five lines clearly show additional flux in the red wing, one is located in a wavelength region too crowded to recognise any asymmetry, and one line is very weak, but looks slightly asymmetric. An example of these asymmetries can be found in Fig. 12 for the double He I line at $7065 \AA$.

We also fitted the two strongest flare-only (quiescent spectrum removed) He I lines with CORA (Ness \& Wichmann 2002). An example of the fit quality can also be found in Fig. 12. We fitted the double line at $7065 \AA$ with two Gaussian components plus one broad Gaussian for the additional line flux in the asymmetry. For each Gaussian the free fit parameters are central wavelength, $F W H M$, and amplitude. The background was fitted manually, since the line is very broad and therefore contributes significantly to the background as fitted by CORA. The same fit procedure was applied to the line at $6678 \AA$, but with the background fitted automatically and only one main component. The fit results can be found in Table 3 .

In the spectrum No. 8, covering the flare onset, the two strongest $\mathrm{He} \mathrm{I}$ lines at 6678 and $7065 \AA$ start to react to the flare. Both lines are blue-shifted and exhibit additional broad, blueshifted components. For the line at $7065 \AA$, we did not find the second narrow component in the data, although this may be due to the low flux in the line. No blue asymmetries can be found in the blue arm of the spectrum, since the blue spectrum No. 2 covering the flare onset is dominated by the continuum and the He I lines are too weak against this strong background.

\subsection{Call lines}

During the flare, we found $9 \mathrm{Ca}$ II lines, out of which 5 are located in the blue arm. Seven lines show additional red wing flux, one is clearly blended with an Fe I line, and another one is so weak that no asymmetry - if present - can be recognised. The Ca II triplet is saturated in the line cores in spectrum No. 9, but the wings are not affected. All three lines show red asymmetries in the wings, although not as strong as the He I lines. We fitted the three lines in the flare-only spectra, again using a narrow and a broad Gaussian, bearing in mind that the narrow component flux is saturated. Nevertheless, the line shifts are unaffected by the saturation. Our line fit parameters are given in Table 4. After spectrum No. 11, the broad component vanishes. In spectrum No. 8, covering the flare onset, no broad component is present. However, all three lines are blue-shifted, although only within 1 to $2 \sigma$ (see Table 4 ).

\subsection{HI lines}

Asymmetries have also been found for the $\mathrm{H} \alpha$ line. During the flare onset in spectrum No. 9, the line has a footpoint width of about $70 \AA$ and the inner wings are also saturated; therefore, we refrained from using this spectrum for the analysis. In the decay phase, the core is saturated for spectra Nos. 10 to 14 , but the wings, where the asymmetries are found, are not affected. We fitted the net-flare line (with the quiescent spectrum No. 1 subtracted) with CORA using two Gaussians, which gives a good empirical description of the line.

The $\mathrm{H} \alpha$ line starts to react to the flare in spectrum No. 8, where it develops broad wings. Moreover, the narrow component is slightly blue-shifted at $6562.71 \pm 0.06 \AA$, whereas the broad component is clearly blue-shifted leading to a blue asymmetry, as can be seen in Fig. 13. The line core (note, that spectrum No. 8 is not saturated) exhibits a self-reversal due to non-LTE effects, which is typical for dMe stars and also reproduced in chromospheric modelling of M dwarfs; see e.g. Cram \& Mullan (1979); Robinson et al. (1990), and Houdebine \& Doyle (1994). The weak asymmetry of the red and the blue peaks also points to the complicated dynamical processes during flares and may be due to a wave propagating downwards. Examples of theoretical $\mathrm{H} \alpha$ profiles from radiative hydrodynamic flare models for $\mathrm{M}$ dwarfs also exhibiting peak asymmetries due to propagating waves can be found in Allred et al. (2006). 
Table 3. Line-fit parameters of the He I line at 7065 and $6678 \AA$.

\begin{tabular}{cccccc}
\hline \hline \multicolumn{5}{c}{ He I at 7065 $\AA^{1}$} \\
No. & $\begin{array}{c}\text { flux narrow 1 } \\
{\left[10^{-16} \mathrm{erg} \mathrm{s}^{-1} \mathrm{~cm}^{-2}\right]}\end{array}$ & $\begin{array}{c}\text { flux narrow 2 } \\
{\left[10^{-16} \mathrm{erg} \mathrm{s}^{-1} \mathrm{~cm}^{-2}\right]}\end{array}$ & $\begin{array}{c}\text { flux broad } \\
{\left[10^{-16} \mathrm{erg} \mathrm{s}^{-1} \mathrm{~cm}^{-2}\right]}\end{array}$ & $\begin{array}{c}F W H M \text { broad } \\
{[\AA]}\end{array}$ & $\begin{array}{c}\text { velocity } \\
\mathrm{km} \mathrm{s}^{-1}\end{array}$ \\
\hline 8 & $1294 \pm 118$ & - & $1212 \pm 192$ & $0.85 \pm 0.09$ & $-3.8 \pm 11.9$ \\
9 & $11143 \pm 206$ & $4139 \pm 223$ & $8844 \pm 442$ & $1.11 \pm 0.03$ & $38.6 \pm 19.5$ \\
10 & $5562 \pm 112$ & $1386 \pm 101$ & $2112 \pm 192$ & $1.04 \pm 0.10$ & $45.8 \pm 13.1$ \\
11 & $2389 \pm 78$ & $437 \pm 62$ & $908 \pm 154$ & $1.13 \pm 0.01$ & $28.0 \pm 19.5$ \\
\multicolumn{5}{c}{ He I at 6678 $\AA^{2}$} \\
\hline 8 & $1646 \pm 145$ & $2235 \pm 217$ & $0.66 \pm 0.03$ & $-10.8 \pm 11.2$ \\
9 & $22360 \pm 295$ & $24583 \pm 467$ & $1.02 \pm 0.01$ & $24.7 \pm 0.5$ \\
10 & $10033 \pm 150$ & $6512 \pm 207$ & $0.91 \pm 0.05$ & $22.4 \pm 2.7$ \\
11 & $3993 \pm 105$ & & $2310 \pm 140$ & $0.68 \pm 0.07$ & $10.8 \pm 14.8$ \\
12 & $2022 \pm 75$ & $610 \pm 110$ & $0.84 \pm 0.08$ & $9.0 \pm 25.1$ \\
13 & $628 \pm 46$ & & & \\
\hline
\end{tabular}

${ }^{1}$ The first narrow component of the line is stable at $7065.19 \AA$, the second is stable at $7065.73 \AA$. Only for spectrum No. 8, the line center is shifted to $7065.10 \pm 0.12 \AA$. The $F W H M$ of both narrow components is $\sim 0.23 \AA$. The background is fitted manually. ${ }^{2}$ The narrow component of the line is stable at $6678.15 \AA$ besides for spectrum No. 8, where it is blue-shifted to $6678.03 \pm 0.10 \AA$.

Table 4. Line shift of the broad component of the Ca II triplet lines from the flare-only spectra.

\begin{tabular}{|c|c|c|c|c|c|c|c|c|c|}
\hline \multirow[b]{2}{*}{ No. } & \multicolumn{3}{|c|}{ Ca II at $8498.018 \AA^{1}$} & \multicolumn{3}{|c|}{ Ca II at $8542.089 \AA^{2}$} & \multicolumn{3}{|c|}{ Ca II at $8662.140 \AA^{3}$} \\
\hline & $\begin{array}{c}\text { position } \\
{[\AA]}\end{array}$ & $\begin{array}{l}\text { velocity } \\
{\left[\mathrm{km} \mathrm{s}^{-1}\right]}\end{array}$ & $\begin{array}{c}F W H M \\
{[\AA]}\end{array}$ & $\begin{array}{c}\text { position } \\
[\AA]]\end{array}$ & $\begin{array}{l}\text { velocity } \\
{\left[\mathrm{km} \mathrm{s}^{-1}\right]}\end{array}$ & $\begin{array}{c}F W H M \\
{[\AA]}\end{array}$ & $\begin{array}{c}\text { position } \\
[\AA]]\end{array}$ & $\begin{array}{l}\text { velocity } \\
{\left[\mathrm{km} \mathrm{s}^{-1}\right]}\end{array}$ & $\begin{array}{c}F W H M \\
{[\AA]}\end{array}$ \\
\hline 9 & $8498.32 \pm 0.02$ & $11.6 \pm 0.7$ & $0.89 \pm 0.04$ & $8542.22 \pm 0.13$ & $5.3 \pm 4.6$ & $1.00 \pm 0.01$ & $8662.33 \pm 0.01$ & $7.3 \pm 0.3$ & $0.91 \pm 0.02$ \\
\hline 10 & $8498.48 \pm 0.10$ & $17.3 \pm 3.5$ & $0.72 \pm 0.02$ & $8542.17 \pm 0.17$ & $3.5 \pm 6.0$ & $0.78 \pm 0.02$ & $8662.23 \pm 0.19$ & $3.8 \pm 6.6$ & 0.70 \\
\hline 11 & $8498.34 \pm 0.43$ & $12.3 \pm 15.2$ & $0.72 \pm 0.02$ & $8542.07 \pm 0.15$ & $0.0 \pm 5.3$ & $0.78 \pm 0.05$ & $8662.22 \pm 0.22$ & $3.4 \pm 7.6$ & 0.70 \\
\hline
\end{tabular}

${ }^{1}$ The narrow component is stable at $8497.99 \pm 0.02 \AA$, besides spectrum No. 8 which is blue-shifted to $8497.80 \pm 0.11 \AA\left(-6.7 \pm 3.9 \mathrm{~km} \mathrm{~s}^{-1}\right)$.

${ }^{2}$ The narrow component is stable at $8542.07 \pm 0.03 \AA$, besides spectrum No. 8 which is blue-shifted to $8541.96 \pm 0.11 \AA\left(-3.8 \pm 3.8 \mathrm{~km} \mathrm{~s} \mathrm{~s}^{-1}\right)$.

${ }^{3}$ The narrow component is stable at $8662.12 \pm 0.04 \AA$, besides spectrum No. 8 which is blue-shifted to $8662.02 \pm 0.07 \AA\left(-3.8 \pm 2.4 \mathrm{~km} \mathrm{~s}^{-1}\right)$.

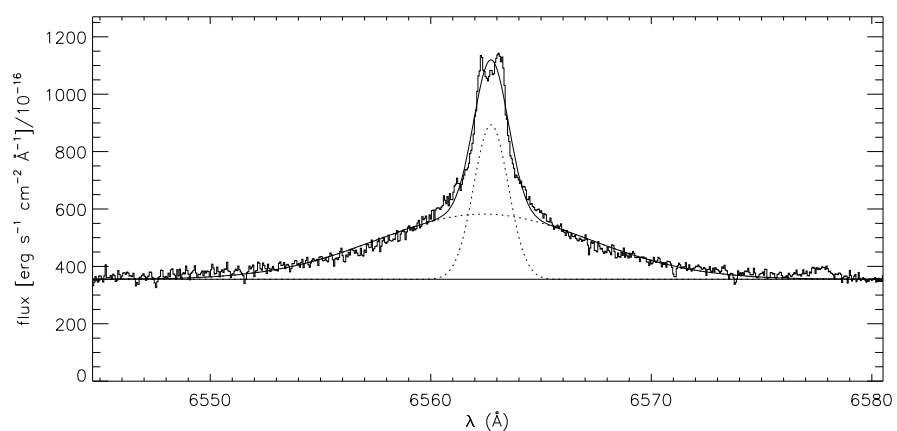

Fig. 13. The $\mathrm{H} \alpha$ line from spectrum No. 8 with the quiescent spectrum subtracted. The line exhibits a clear blue asymmetry due to the blue-shifted broad component. The narrow component is also slightly blue-shifted.

The broad component is red-shifted in the spectra covering the flare decay phase. The derived wavelength shifts are listed in Table 5, assuming a rest wavelength of $6562.817 \AA$ (Moore 1972). The broad component has vanished in spectrum No. 15.

Besides the $\mathrm{H} \alpha$ line, we also analysed the $\mathrm{H}_{9}$ line in detail. For the even higher Balmer lines, we refrained from a detailed analysis, since the broad wings of these lines have to be deblended from metal lines, Due to decreasing flux this becomes more and more difficult for higher Balmer lines.

We performed a simultaneous fit with three metal lines for the $\mathrm{H}_{9}$ line, i.e. a 5-component Gaussian fit with three Gaussians for the metal lines and one narrow and one broad Gaussian for the $\mathrm{H}_{9}$ line itself. This procedure led to a very good description
Table 5. Line parameters for the broad component of the $\mathrm{H} \alpha$ line. Note, that there is no measurement for spectrum No. 9.

\begin{tabular}{cccc}
\hline \hline No. & $\begin{array}{c}\text { Position } \\
{[\AA]}\end{array}$ & $\begin{array}{c}\text { Velocity } \\
{\left[\mathrm{km} \mathrm{s}^{-1}\right]}\end{array}$ & $\begin{array}{c}F W H M \\
{[\AA]}\end{array}$ \\
\hline 8 & $6562.47 \pm 0.10$ & $-15.8 \pm 4.6$ & $4.82 \pm 0.02$ \\
10 & $6563.39 \pm 0.01$ & $26.2 \pm 0.5$ & $4.10 \pm 0.01$ \\
11 & $6563.08 \pm 0.01$ & $12.0 \pm 0.5$ & $2.90 \pm 0.01$ \\
12 & $6563.21 \pm 0.01$ & $17.9 \pm 0.5$ & $2.12 \pm 0.02$ \\
13 & $6563.60 \pm 0.06$ & $35.8 \pm 2.7$ & $1.50 \pm 0.09$ \\
14 & $6562.88 \pm 0.38$ & $2.9 \pm 17.4$ & $3.10 \pm 0.11$ \\
\hline
\end{tabular}

of the data, except for spectra Nos. 2 and 3. For these two spectra, we changed the narrow Gaussian profile to a Lorentzian profile again leading to acceptable fits. For spectrum No. 2, which covers the flare onset, a weak blue asymmetry could be detected with the broad component at $3835.34 \pm 0.03 \AA$. The Lorentzian describing the narrow component is only blue-shifted within the error bars: $3835.36 \pm 0.08 \AA$. The $F W H M$ is $0.51 \AA$ for the Lorentzian and $2.20 \AA$ for the broad Gaussian. During the flare peak spectrum No. 3, the broad component is red-shifted at $3835.47 \pm 0.01 \AA$ with a $F W H M$ of $3.34 \AA$. The following spectra tend to have red-shifted broad components, but within the large errors. Nevertheless, no line shift in the consecutive spectra agrees with what we found for $\mathrm{H} \alpha$, where the broad component vanishes after spectrum No. 14 (cf., Fig. 1).

In addition to the Balmer lines, we searched the Paschen lines for asymmetries, but none were found; however, since these 
lines are very broad and noisy, an asymmetry could easily be hidden in the data (see Fig. 9).

\subsection{Discussion of the line asymmetries}

We measured line asymmetries for He II, Ca II, and Balmer lines during the flare, but not for the Paschen lines and other metal lines (although the Mg II lines also may exhibit small asymmetries). While in the case of the Paschen lines this can be attributed to the enormous line widths hiding asymmetries, this argument is not valid for the metal lines. However, the lines of the abovementioned ions tend to form at different chromospheric heights (and therefore at different temperatures) than the rest of the observed metal lines. While the line cores of the above-mentioned ions tend to form in the upper chromosphere or transition region onset, the line cores of the other metals tend to form in the lower to mid chromosphere (Vernazza et al. 1981; Fuhrmeister et al. 2005b). This gives a hint at least to the temperature of the moving material, which we ascribe the asymmetries to. The material must be rather hot (about 8000 to $10000 \mathrm{~K}$ ) compared to the rest of the chromosphere. We also ascribe the non-uniform reactions of the asymmetries of the different lines to different line formation conditions. Nevertheless, trends in the reaction of the lines can be noticed: in our spectra, we find asymmetries in the blue as well as in the red wings. Asymmetries on the blue side are found during flare onset, red wing asymmetries during decay phase. This makes the interpretation as moving material very straightforward. At the flare onset, the chromospheric evaporation is seen as a blue shift (although partly only within the errors) of the main/narrow components of the analysed strong lines. In addition, there seems to be a rising "cloud" of material, but moving with a different velocity producing the broad component. The main/narrow line component does not indicate any moving material in the decay phase, but the "cloud" is now moving downward, leading to the observed red asymmetries. For the $\mathrm{H} \alpha$ line, we detected not only a decay of the asymmetry, but also variations in the asymmetry from spectrum to spectrum, favouring a scenario of not one single uniform downflowing "cloud", but of a multitude of downflows. These are de-accelerated when hitting deeper and denser atmospheric layers, which is seen in the generally decreasing velocity of the broad component with evolution of the flare.

During the decay phase, downflow velocities range from $\sim 5$ to $40 \mathrm{~km} \mathrm{~s}^{-1}$, which is probably supersonic. The sound velocity in models of quiescent chromospheres is about $10 \mathrm{~km} \mathrm{~s}^{-1}$ (Jevremović et al. 2000). Similar downflow velocities have been measured for the flare on LHS 2034 (Fuhrmeister et al. 2005a), but downflow velocities of up to $60 \mathrm{~km} \mathrm{~s}^{-1}$ are normally observed for the Sun (Fisher 1989).

Interpretating the $F W H M$ of the broad component is difficult because it may have different components. At least for the hydrogen lines, Stark broadening should contribute to the line width, and turbulent motions are also a possible interpretation. Since there may be different downflow kernels, the width of the line may be due to the different bulk motions of these kernels that are integrated in our spectra.

In conclusion, we tentatively interpret our observations in a scenario of a chromospheric prominence that is lifted during the flare onset and then raining down during the decay phase.

\section{Discussion and conclusion}

We presented the optical part of a multiwavelength campaign that caught $\mathrm{CN}$ Leo in a spectacular flare. There are only few observations of such giant flares, but even these few examples show very different timescales and lightcurve characteristics. While the CN Leo flare lasted about 50 min, a 5-mag flare in the $U$-Band on UV Cet lasted only 12 min (Eason et al. 1992), while the large flare on AD Leo from 1985 lasted more than $4 \mathrm{~h}$ (Hawley \& Pettersen 1991). Compared to somewhat smaller (but nevertheless very large) flares, these can be of the same duration or even longer than the CN Leo flare. For example, a $2.1 \mathrm{mag}$ flare in the $U$-band was observed on AD Leo to last about $50 \mathrm{~min}$ (Houdebine et al. 1993), while a large flare on AT Mic lasted about $1.5 \mathrm{~h}$ (García-Alvarez et al. 2002).

Most of the large flares show a double (or triple) peaked lightcurve in the $U$-band and also for Balmer emission lines like the CN Leo flare (Hawley \& Pettersen 1991; Houdebine et al. 1993; García-Alvarez et al. 2002). The UV Cet flare had an even more complicated substructure with at least 4 peaks in the major outburst (Eason et al. 1992). This multiple-peak flare pattern is usually interpreted in terms of different reconnection events, which may trigger each other in succession. The CN Leo flare shows two major peaks that can be easily noticed in the exposuremeter data in Fig. 1, with a minor peak between the two. The time between the two major peaks is about $200 \mathrm{~s}$. For the 2.1 mag flare on AD Leo, the two major peaks are separated by about 9 min (Houdebine et al. 1993). For all other mentioned flares (besides UV Cet), the separation is even longer.

The flux peaks of chromospheric lines are typically delayed with respect to the $U$-band flux peak, with the $\mathrm{Ca}$ II $\mathrm{H}$ and $\mathrm{K}$ lines even more delayed than the Balmer lines (e.g. García-Alvarez et al. 2002; Hawley \& Pettersen 1991). The time resolution of our spectra is unfortunately too low to observe any delay in the peak, but as is apparent in Fig. 8, the flux of the Ca II triplet line decays more slowly than the other lines. This behaviour is compatible with what is normally observed for the Ca II $\mathrm{H}$ and $\mathrm{K}$ lines, which decay more gradually than Balmer and Helium lines (e.g. Houdebine et al. 1993), consistent with an interpretation of a faster evolution of high-temperature lines in the chromosphere (Houdebine 2003).

The optical spectral data allowed us to trace the chromospheric reaction to the flare (with one He II transition line covered). We found a wealth of chromospheric emission lines that has not been observed before, especially in the red part of the spectrum; also, V II and Sc II lines show up in the blue arm. Especially in the flare onset spectrum No. 2, the higher Balmer lines develop very broad damping wings that can only be fitted involving a Lorentzian profile. With respect to line shapes and profiles, we find asymmetries varying from blue excess flux during flare onset to red excess flux during the decay phase. In the red excess for $\mathrm{H} \alpha$, not only a general decay is found, but also variations from spectrum to spectrum, providing strong evidence for a scenario involving multiple flare kernels, each triggering chromospheric downward condensations. We ascribe the blue excess flux during flare.

The optical quasi-continuum is also quite massively affected by the flare. The blue spectrum is clearly blackbody-dominated during flare onset. The same is true for the red spectrum with the quiescent spectrum removed. The derived temperatures for the gas producing the blackbody radiation in the first few seconds of the flare onset exceed of $20000 \mathrm{~K}$ and then rapidly cool.

This set of excellent optical data, which characterises the photosphere and chromosphere of CN Leo during a giant flare is suited well to classical semi-empirical chromospheric flare modelling with a stellar atmosphere code. Modelling using a multitude of emission lines will be presented in a forthcoming paper in this series (Fuhrmeister et al. 2008). 
Acknowledgements. B.F. and C.L. acknowledge financial support by the DLR under 50OR0105. A.R. has received research funding from the DFG as an Emmy Noether Fellow (DFG 1664/4-1).

\section{References}

Allred, J. C., Hawley, S. L., Abbett, W. P., \& Carlsson, M. 2006, ApJ, 644, 484 Berger, E., Rutledge, R. E., Reid, I. N., et al. 2005, ApJ, 627, 960

Berger, E., Basri, G., Gizis, J. E., et al. 2008a, ApJ, 676, 1307

Berger, E., Gizis, J. E., Giampapa, M. S., et al. 2008b, ApJ, 673, 1080

Canfield, R. C., Penn, M. J., Wulser, J.-P., \& Kiplinger, A. L. 1990, ApJ, 363, 318

Cram, L. E., \& Mullan, D. J. 1979, ApJ, 234, 579

Crespo-Chacón, I., Montes, D., García-Alvarez, D., et al. 2006, A\&A, 452, 987

de Jager, C., Heise, J., van Genderen, A. M., et al. 1989, A\&A, 211, 157

Doyle, J. G., Butler, C. J., Bryne, P. B., \& van den Oord, G. H. J. 1988, A\&A, 193,229

Eason, E. L. E., Giampapa, M. S., Radick, R. R., Worden, S. P., \& Hege, E. K. 1992, AJ, 104, 1161

Fisher, G. H. 1989, ApJ, 346, 1019

Fuhrmeister, B., Schmitt, J. H. M. M., \& Hauschildt, P. H. 2005a, A\&A, 436, 677

Fuhrmeister, B., Schmitt, J. H. M. M., \& Hauschildt, P. H. 2005b, A\&A, 439, 1137

Fuhrmeister, B., Liefke, C., \& Schmitt, J. H. M. M. 2007, A\&A, 468, 221

Fuhrmeister, B., Schmitt, J. H. M. M., \& Hauschildt, P. H. 2008, A\&A, in preparation

García-Alvarez, D., Jevremović, D., Doyle, J. G., \& Butler, C. J. 2002, A\&A, 383,548

Güdel, M., Audard, M., Skinner, S. L., \& Horvath, M. I. 2002, ApJ, 580, L73

Güdel, M., Audard, M., Reale, F., Skinner, S. L., \& Linsky, J. L. 2004, A\&A, 416,713
Gunn, A. G., Doyle, J. G., Mathioudakis, M., Houdebine, E. R., \& Avgoloupis, S. 1994, A\&A, 285, 489

Hawley, S. L., \& Fisher, G. H. 1992, ApJS, 78, 565

Hawley, S. L., \& Pettersen, B. R. 1991, ApJ, 378, 725

Hawley, S. L., Allred, J. C., Johns-Krull, C. M., et al. 2003, ApJ, 597, 535

Houdebine, E. R. 2003, A\&A, 397, 1019

Houdebine, E. R., \& Doyle, J. G. 1994, A\&A, 289, 169

Houdebine, E. R., Foing, B. H., Doyle, J. G., \& Rodono, M. 1993, A\&A, 274, 245

Jevremović, D., Doyle, J. G., \& Short, C. I. 2000, A\&A, 358, 575

Kirkpatrick, J. D., Henry, T. J., \& McCarthy, D. W. 1991, ApJS, 77, 417

Liebert, J., Kirkpatrick, J. D., Reid, I. N., \& Fisher, M. D. 1999, ApJ, 519, 345

Liefke, C., Schmitt, J. H. M. M., \& Fuhrmeister, B. 2008, A\&A, in preparation

Mitra-Kraev, U., Harra, L. K., Güdel, M., et al. 2005, A\&A, 431, 679

Moore, C. E. 1972, Nat. Stand. Ref. Data Ser., 40

Ness, J.-U., \& Wichmann, R. 2002, Astron. Nachr., 323, 129

Osten, R. A., Hawley, S. L., Allred, J. C., Johns-Krull, C. M., \& Roark, C. 2005, ApJ, 621, 398

Osten, R. A., Hawley, S. L., Allred, J., et al. 2006, ApJ, 647, 1349

Paulson, D. B., Allred, J. C., Anderson, R. B., et al. 2006, PASP, 118, 227

Pavlenko, Y. V., Jones, H. R. A., Lyubchik, Y., Tennyson, J., \& Pinfield, D. J. 2006, A\&A, 447, 709

Piskunov, N. E., \& Valenti, J. A. 2002, A\&A, 385, 1095

Reid, I. N., Hawley, S. L., \& Gizis, J. E. 1995, AJ, 110, 1838

Reiners, A., Schmitt, J. H. M. M., \& Liefke, C. 2007, A\&A, 466, L13

Robinson, R. D., Cram, L. E., \& Giampapa, M. S. 1990, ApJS, 74, 891

Schmidt, S. J., Cruz, K. L., Bongiorno, B. J., Liebert, J., \& Reid, I. N. 2007, AJ, 133,2258

Schmitt, J. H. M. M., Reale, F., Liefke, C., et al. 2008, A\&A, 481, 799

Smith, K., Güdel, M., \& Audard, M. 2005, A\&A, 436, 241

Vernazza, J. E., Avrett, E. H., \& Loeser, R. 1981, ApJS, 45, 635 